\title{
Buffer Tank Design for Acceptable Control Performance
}

\author{
Audun Faanes* and Sigurd Skogestad ${ }^{\dagger}$ \\ Department of Chemical Engineering \\ Norwegian University of Science and Technology \\ N-7491 Trondheim, Norway
}

April 4, 2003

Ind. Eng. Chem. Res.

(in press)

\begin{abstract}
This paper provides a systematic approach for the design of buffer tanks. We consider mainly the case where the objective of the buffer tank is to dampen ("average out") the fast (i.e., high-frequency) disturbances, which cannot be handled by the feedback control system. We consider separately design procedures for (I) mixing tanks to dampen quality disturbances and (II) surge tanks with averaging level control to handle flow-rate disturbances.
\end{abstract}

*also affiliated with Norsk Hydro ASA, Corporate Research Centre, N-3907 Porsgrunn, Norway, E-mail: audun.faanes@hydro.com, Tel.: +47 359240 21, Fax.: +47 35923263

${ }^{\dagger}$ Author to whom all correspondence should be addressed. E-mail: skoge@ chemeng.ntnu.no, Tel.: +47 7359 4154 , Fax.: +47 73594080 


\section{Introduction}

Buffer tanks are common in industry, under many different names, such as intermediate storage vessels, holdup tanks, surge drums, accumulators, inventories, mixing tanks, continuous stirred tank reactors (CSTRs), and neutralization vessels. We start with a definition:

\section{A buffer tank is a unit where the holdup (volume) is exploited to provide smoother operation.}

We here focus on buffer tanks for liquids, although most of the results may be easily extended to gas- or solid-phase systems. Buffer tanks may be divided into two categories, namely, for (A) disturbance attenuation and (B) independent operation:

A. Buffer tanks are installed between units to avoid propagation of disturbances for continuous processes.

B. Buffer tanks are installed between units to allow independent operation, for example during a temporary shutdown and between continuous and batch process units.

In this category there is a continuous delivery or outdraw on one side and a discontinuous delivery or outdraw on the other side. The design of the tank size for these types of buffer tanks is often fairly straightforward (typically equal to the batch volume) and is not covered further in this paper.

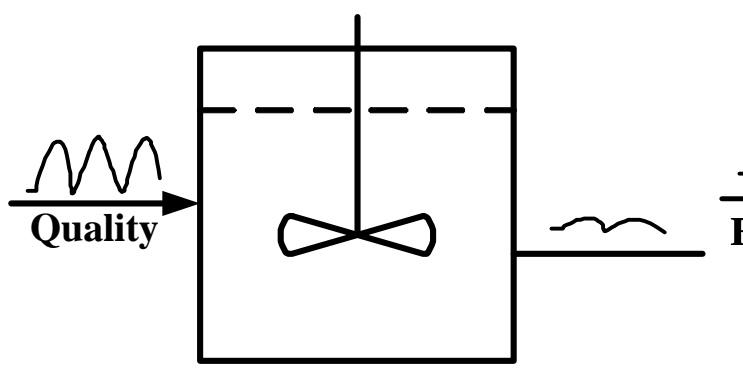

(I) Averaging by mixing (mixing tank)

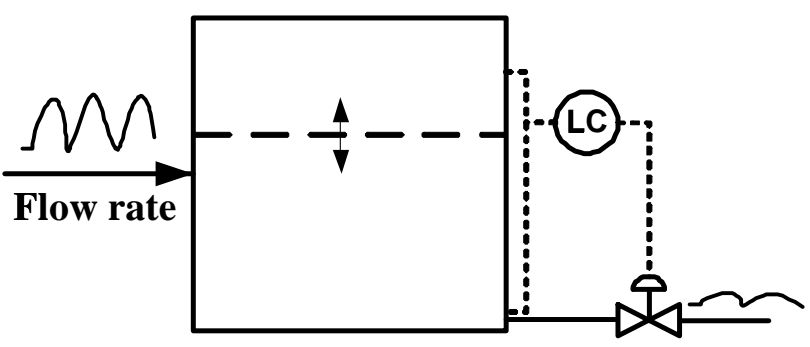

(II) Averaging level control (surge tank)

Figure 1: Two types of buffer tanks

In this paper we focus on category A. There are two fundamentally different disturbances, namely, in quality and flow rate, and two approaches to dampen them (see Figure 1):

I. Quality disturbances, e.g., in concentration or temperature, where we dampen by mixing. Such buffer tanks are often called mixing tanks or neutralization vessels for $\mathrm{pH}$ processes.

II. Flow-rate disturbances, e.g., in the feed rate, where we dampen by temporarily changing the volume (level variation). Such buffer tanks are often called surge tanks, intermediate storage vessels, holdup tanks, surge drums, accumulators, or inventories. 
In both cases the tank volume is exploited, and a larger volume gives better dampening: In the first case, mixing of a larger volume means that the in-flow entering during a longer period is mixed together, and in the second case, larger level variations are allowed.

Often, in the design of buffer tanks, the residence or hold-up time is used as a measure instead of the volume. The residence time is defined as $\tau=V / q$, where $V$ is the volume $\left[\mathrm{m}^{3}\right]$ and $q$ the nominal flow rate $\left[\mathrm{m}^{3} / \mathrm{s}\right]$.

Even if the buffer tanks are designed and implemented for control purposes, control theory is rarely used when sizing and designing the tanks. Instead, rules of thumb are used. For example, textbooks on chemical process design seem to agree that a half-full residence time of 5-10 minutes is appropriate for distillation reflux drums and that this also applies for many other buffer (surge) tanks. For tanks between distillation columns, a half-full residence time of 10-20 minutes is recommended (Lieberman, 1983; Sandler and Luckiewicz, 1987; Ulrich, 1984; Walas, 1987; Wells, 1986).

Sigales (1975) sets the total residence time as the sum of the surge time and a possible settling time. The following surge times are recommended: distillation reflux, 5 minutes; product to storage, 2 minutes; product to heat exchanger or other process streams, 5 minutes; product to heater, 10 minutes. The settling time applies when there is an extra liquid phase. For water in hydrocarbons, a settling time of 5 minutes is proposed.

None of the above references provide any justifications for their rules.

The most complete design procedure for reflux drum volumes is presented by Watkins (1967), who proposes a half-full volume given by

$$
V=\left(f_{1}+f_{2}\right)\left(L+f_{3} D\right) f_{4}
$$

Here $f_{1}$ (typical range 0.5-2) and $f_{2}$ (typical range 1-2) are instrumentation and labor factors, respectively, related to buffer tanks of category $\mathrm{B}$ mentioned above. For example, the value of $f_{2}$ may be based on how much time it takes for the operator to replace a disabled pump. $L$ and $D$ are reflux and product rates, and the factor $f_{3}$ (typical range 1.25-4) is dependent on how well external units are operated (e.g., 1.25 for product to storage). $f_{4}$ (typical range 1-2) is a level indicator factor. The method gives half-full hold-up times from 1.5 to $32 \mathrm{~min}$.

In addition to the volumes proposed above, one normally adds about $10 \%$ of the volume to prevent overfilling (Wells, 1986). For reflux drums, 25-50\% extra volume for the vapor is recommended (Sandler and Luckiewicz, 1987).

A basic guide to the design of mixing tanks is given by (Ludwig, 1977).

The process control literature refers to the level control of buffer tanks for flow-rate dampening (surge tanks) as averaging level control. Harriott (1964), Hiester et al. (1987), and Marlin (1995) propose controller and tank size designs that are based on specifying the maximum allowed change in the flow rate out of the buffer (surge) tank because this flow acts as a disturbance for the downstream process. However, no guidelines are given for the critical step of specifying the outlet flow-rate change. Otherwise, these methods have similarities with the one proposed in the present paper.

To reduce the effect of the material balance control on the quality control loop, Buckley (1964) recommends designing the buffer tank such that the material balance control can be made 10 times slower than the quality loop. In practice, this means that the effect of the disturbance on the quality at the worst-case frequency is reduced by a factor of 10 . This applies to both surge and mixing tanks.

There have also been proposals for optimal averaging level control, e.g., (McDonald et al., 1986), where the objective is to find the controller that essentially gives the best disturbance dampening for a given surge tank. To reduce the required surge tank volume, provided one 
is willing to accept rare and short large changes in the outlet flow, one may use a nonlinear controller that works as an averaging controller when the flow changes are small but where the nonlinear part prevents the tank from being completely empty or full, e.g., (McDonald et al., 1986; Shunta and Fehervari, 1976; Shinskey, 1996).

Another related class of process equipment is neutralization tanks. Neutralization is a mixing process of two or more liquids of different $\mathrm{pH}$. Normally this takes place in one or more buffer (mixing) tanks in order to dampen variations in the final product. The process design for neutralization is discussed by Shinskey (1973) and McMillan (1984). Another design method and a critical review on the design and control of neutralization processes with emphasis on chemical wastewater treatment is found in Walsh (1993). In (Faanes and Skogestad, 2002) tank size selection for neutralization processes is discussed.

Zheng and Mahajanam (1999) propose the use of the necessary buffer tank volume as a controllability measure.

The objective of this paper is to answer the following questions: When should a buffer tank be installed to avoid propagation of disturbances, and how large should the tank be? The preferred way of dealing with disturbances is feedback control. Typically, with integral feedback control, perfect compensation may be achieved at steady state. However, because of inherent limitations such as time delays, the control system is generally not effective at higher frequencies, and the process itself (including any possible buffer tanks) must dampen high-frequency disturbances. We have the following:

The buffer tank (with transfer function $h(s)$ ) should modify the disturbance, $d$, such that the modified disturbance

$$
d^{\prime}(s)=h(s) d(s)
$$

can be handled by the control system. The buffer tank design problem can be solved in two steps:

Step 1. Find the required transfer function $h(s)$. (Typically $h(s)=1 /(\tau s+1)^{n}$, and the task is to find the order $n$ and the time constant $\tau$.)

Step 2. Find a physical realization of $h(s)$ (tank volume $V$ and possibly level control tuning).

In this paper we present design methods for buffer tanks based on this fundamental insight.

\section{Introductory example}

The following example illustrates how we may use (1) the control system and (2) a buffer (mixing) tank to keep the output within its specified limits despite disturbances.

Example 1 Consider the mixing of two process streams, $A$ and $B$, with different components (also denoted $A$ and $B$ ), as illustrated in Figure 2.

The objective is to mix equal amounts of $A$ and $B$ such that the excess concentration of the outlet flow $c_{0}=c_{A}-c_{B}$ is close to zero. More specifically, we require $c_{0}$ to stay within $0 \pm 1 \mathrm{~mol} / \mathrm{m}^{3}$. The combined component and total material balance gives the following model:

$$
\frac{d c_{0}}{d t}=\frac{1}{V}\left[\left(c_{A, \mathrm{f}}-c_{0}\right) q_{A}+\left(c_{B, \mathrm{f}}-c_{0}\right) q_{B}\right]
$$




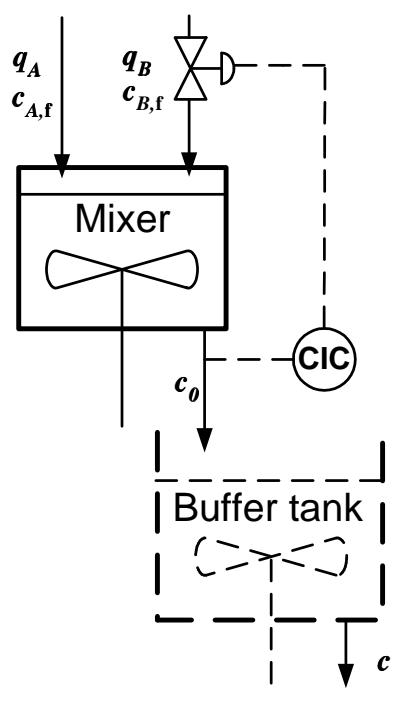

Figure 2: Mixing process. The concentration is controlled by manipulating the flow rate of stream $B$. Variations are further dampened by an extra buffer tank.

For the case with no control and no buffer tank, the time response in the outlet concentration, $y=c_{0}$, to a step disturbance in the feed concentration, $d=c_{A, \mathrm{f}}$, is shown by the solid line ("Original") in Figure 3. The value of $y=c_{0}$ approaches $10 \mathrm{~mol} / \mathrm{m}^{3}$, which is 10 times larger than the accepted value.

1. We first design a feedback control system, based on measuring $y=c_{0}$, and manipulating $u=q_{B}$ to counteract the disturbance. We choose a proportional-integral (PI) composition controller, $K_{C I C}(s)=0.01(s+1) / s$. Note that the speed of the control system is limited by an effective delay $\theta=1 \mathrm{~min}$, mainly due to the concentration measurement. The resulting response with control is shown by the dashed line. Because the controller has integral action, the outlet concentration returns to its desired value of $0 \mathrm{~mol} / \mathrm{m}^{3}$. However, because of the delay, the initial deviation is still unacceptable.

2. To deal with this, we install, in addition, a buffer tank with volume $19 \mathrm{~m}^{3}$ (residence time $19 \mathrm{~min}$ ) (drawn with dashed lines in Figure 2). We are now able to keep the outlet concentration $c$ within its limit of $\pm 1 \mathrm{~mol} / \mathrm{m}^{3}$ at all times as shown by the dash-dotted line in Figure 3.

Instead of the buffer tank, we could have installed a feedforward controller, but this requires a fast (and accurate) measurement of the disturbance, $d=c_{A, f}$, and a good process model. In practice, it would be very difficult to make this work for this example.

Comment on notation: Throughout the paper, the main feedback controller for the process is denoted $K(s)$, whereas the buffer tank level controller is denoted $k(s)$.

In the following sections we will show how to design buffer tanks for quality disturbances, like in the above example, as well as for flow-rate disturbances. 


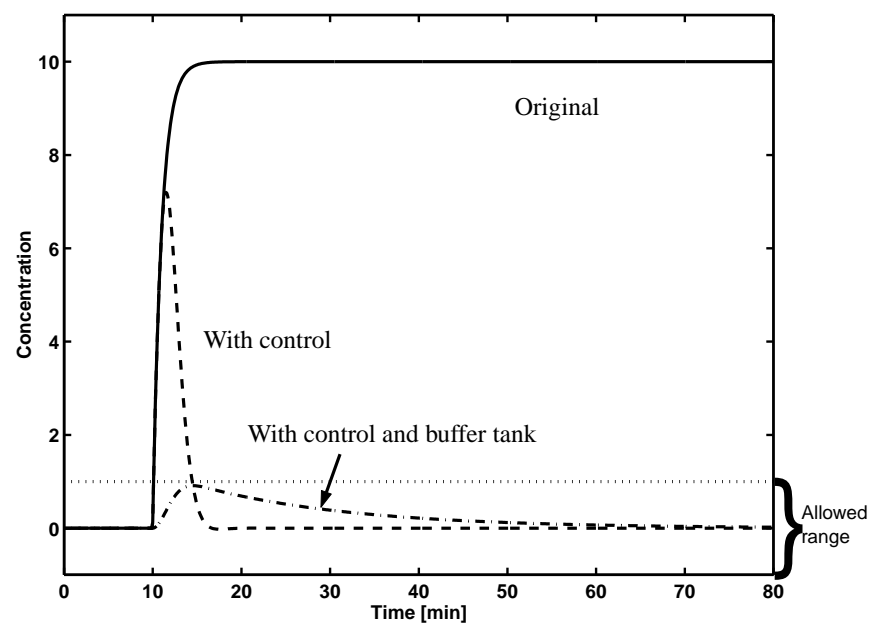

Figure 3: Response in the excess outlet concentration to a step in inlet quality (from $100 \mathrm{~mol} \mathrm{~A} / \mathrm{m}^{3}$ to $120 \mathrm{~mol} A / \mathrm{m}^{3}$ at $10 \mathrm{~min}$ ) for the system in Figure 2. A composition controller handles the long term ("slow") disturbance, but a buffer tank is required to handle the short term deviations. Nominal data: $q_{A}=0.5 \mathrm{~m}^{3} / \mathrm{min}, q_{B}=0.5 \mathrm{~m}^{3} / \mathrm{min}, c_{A}=100 \mathrm{~mol} \mathrm{~A} / \mathrm{m}^{3}$, $c_{B}=100 \mathrm{~mol} B / \mathrm{m}^{3}, c_{0}=0 \mathrm{~mol} A-B / \mathrm{m}^{3}$. Residence time mixer: $1 \mathrm{~min}$. Delay in control loop $\theta=1 \mathrm{~min}$. The levels in the mixer and the buffer tank are controlled by adjusting the outflow with PI controllers, $k(s)=(50 s+1) /(50 s)$.

\section{Step 2: Physical realization of $h(s)$ with a buffer tank}

Consider the effect of a disturbance, $d$, on the controlled variable $y$. Without any buffer tank, the linearized model in terms of deviation variables may be written as

$$
y(s)=G_{d_{0}}(s) d(s)
$$

where $G_{d_{0}}$ is the original disturbance transfer function (without a buffer tank). To illustrate the effect of the buffer tank, we let $h(s)$ denote the transfer function for the buffer tank. The disturbance passes through the buffer tank. With a buffer tank, the model becomes (see Figure 4)

$$
y(s)=\underbrace{G_{d_{0}}(s) h(s)}_{G_{d}(s)} d(s)
$$

where $G_{d}(s)$ is the resulting modified disturbance transfer function. A typical buffer tank transfer function is

$$
h(s)=\frac{1}{(\tau s+1)^{n}}
$$

Note that $h(0)=1$ so that the buffer tank has no steady-state effect.

We will now consider separately how transfer functions $h(s)$ of the form (6) arise for (I) quality and (II) flow-rate disturbances. In both cases, we consider a buffer tank with liquid volume $V\left[\mathrm{~m}^{3}\right]$, inlet flow rate $q_{i n}\left[\mathrm{~m}^{3} / \mathrm{s}\right]$, and outlet flow rate $q\left[\mathrm{~m}^{3} / \mathrm{s}\right]$. 


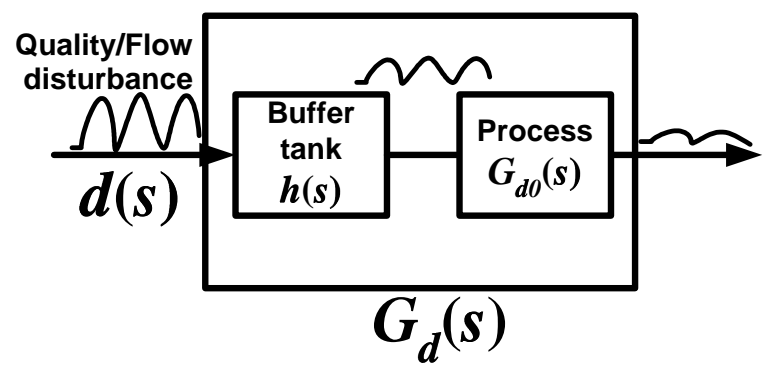

Figure 4: Use of buffer tank to dampen the disturbance

\section{Mixing tank for quality disturbance $\left(d=c_{i n}\right)$}

Let $c_{i n}$ denote the inlet quality and $c$ the outlet quality (for example, concentration or temperature). For quality disturbances, the objective of the buffer tank is to smoothen the quality response

$$
c(s)=h(s) c_{i n}(s)
$$

so that the variations in $c$ are smaller than those in $c_{i n}$. A component or simplified energy balance for a single perfectly mixed tank yields $d(V c) / d t=q_{i n} c_{i n}-q c$. By combining this with the total material balance $d V / d t=q_{i n}-q$ (assuming constant density), we obtain $V d c / d t=q_{i n}\left(c_{i n}-c\right)$, which upon linearization and taking the Laplace transform yields

$$
c(s)=\frac{1}{\frac{V^{*}}{q^{*}} s+1}\left[c_{i n}(s)+\frac{c_{i n}^{*}-c^{*}}{q^{*}} q_{i n}(s)-\frac{c_{i n}^{*}-c^{*}}{V^{*}} V(s)\right]
$$

where an asterisk denotes the nominal (steady-state) values and the Laplace variables $c(s)$, $c_{i n}(s), q_{i n}(s)$, and $V(s)$ now denote deviations from the nominal values. We note that flowrate disturbances (in $q_{i n}$ ) may result in quality disturbances if we mix streams of different compositions (so that $c_{i n}^{*} \neq c^{*}$ ). From (8), we find that the transfer function for the tank is

$$
h(s)=\frac{1}{\tau s+1}
$$

where $\tau=V^{*} / q^{*}[\mathrm{~s}]$ is the nominal residence time. We note that the buffer (mixing) tank works as a first-order filter. Similarly, for $n$ tanks in series, we have

$$
h(s)=\frac{1}{\prod_{i=1}^{n}\left(\tau_{i} s+1\right)}
$$

where $\tau_{i}=$ residence time in tank $i$. We find the required volume of each tank from $V_{i}=\tau_{i} q_{i}^{*}$, where $q_{i}^{*}$ is the nominal flow rate through tank $i$.

\section{Surge tank for flow-rate disturbance $\left(d=q_{i n}\right)$}

For flow-rate disturbances, the objective is to use the buffer volume to smoothen the flowrate response

$$
q(s)=h(s) q_{i n}(s)
$$


The total mass balance assuming constant density yields

$$
\frac{d V}{d t}=q_{i n}-q
$$

We want to use an "averaging level control" with a "slow" level controller, because tight level control yields $d V / d t \approx 0$ and $q \approx q_{i n}$. Let $k(s)$ denote the transfer function for the level controller including measurement and actuator dynamics and also the possible dynamics of an inner flow control loop. Then

$$
q(s)=k(s)\left(V(s)-V_{s}(s)\right)
$$

where $V_{s}$ is the set-point for the volume. Combining this with (12) and taking Laplace transforms yields

$$
V(s)=\frac{1}{s+k(s)}\left[q_{i n}(s)+k(s) V_{s}(s)\right]
$$

or from (13):

$$
q(s)=\frac{k(s)}{s+k(s)}\left[q_{i n}(s)-s V_{s}(s)\right]
$$

The buffer (surge) tank transfer function is thus given by

$$
h(s)=\frac{k(s)}{s+k(s)}=\frac{1}{\frac{s}{k(s)}+1}
$$

With a proportional controller, $k(s)=k_{c}$, we get that $h(s)$ is a first-order filter with $\tau=1 / k_{c}$. Alternatively, for a given $h(s)$, the resulting controller is

$$
k(s)=\frac{s h(s)}{1-h(s)}
$$

Compared to the quality disturbance case, we have more freedom in selecting $h(s)$, because we can quite freely select the controller $k(s)$. However, the liquid level will vary, so the size of the tank must be chosen so that the level remains between its limits. The volume variation is given by (14), which upon combination with (17) yields

$$
V(s)=\frac{1-h(s)}{s} q_{i n}(s)
$$

Note that $V(s)$ represents the deviation from the nominal volume. The maximum value of this transfer function occurs for all of our cases at low frequencies $(s=0)$.

In Table 1 we have found the level controller $k(s)$ and computed the required total volume for $h(s)=1 /(\tau s+1)^{n}$. For example, for a first-order filter, $h(s)=1 /(\tau s+1)$, the required controller is a $\mathrm{P}$ controller with gain $1 / \tau$ and the required volume of the tank is $V_{\text {tot }}=\tau \Delta q_{\max }$.

Note that the resulting level controllers, $k(s)$, do not have integral action. A level controller without integral action was also recommended and further discussed by Buckley (1964, page 167) and Shinskey (1996, page 25).

For flow-rate disturbances, a high-order $h(s)$ can alternatively be realized using multiple tanks with a $\mathrm{P}$ level controller, $k(s)$, in each tank. However, the required total volume is the 
Table 1: Averaging level control: Design procedure II for flow-rate disturbances for alternative choices of $h(s)$.

\begin{tabular}{|l|l|l|l|}
\hline Step & 1st order & 2nd order & nth order \\
\hline 2.1. Desired $h(s)$ (from Step 1) & $\frac{1}{\tau s+1}$ & $\frac{1}{(\tau s+1)^{2}}$ & $\frac{1}{(\tau s+1)^{n}}$ \\
\hline 2.2. Level controller, $k(s)$ from $(17)$ & $1 / \tau$ & $\frac{1}{2 \tau} \frac{1}{\frac{\tau}{2} s+1}$ & $\frac{s}{(\tau s+1)^{n}-1}$ \\
\hline 2.3. $V(0) / q_{\text {in }}(0)$ from $(18)$ & $\tau$ & $2 \tau$ & $n \tau$ \\
\hline 2.4. $V_{\text {tot }}$ & $\tau \Delta q_{\max }$ & $2 \tau \Delta q_{\max }$ & $n \tau \Delta q_{\max }$ \\
\hline
\end{tabular}

same as that found above with a single tank and a more complex $k(s)$, so the latter is most likely preferable from an economic point of view.

\section{Step 1: Desired buffer transfer function $h(s)$}

What is a desirable transfer function, $h(s)$ ? We here present a frequency-domain approach for answering this question. Figure 5 shows the frequency plot of $h(s)=1 /\left(\left(\tau_{h} / n\right) s+1\right)^{n}$ for $n=1$ to 4 , where $\tau_{h}$ in most cases is the total residence time in the tanks. With a given value of $\tau_{h}$, we see that $n=1$ is "best" if we want to reduce the effect of the disturbance at a given frequency by a factor $f=3(=1 / 0.33)$ or less; $n=2$ is "best" if the factor is between 3 and about $7(=1 / 0.144)$, and $n=3$ is "best" if the factor is between about 7 and $15(=1 / 0.064)$. Thus, we find that a larger order $n$ is desired when we want a large disturbance reduction. We now derive more exactly the desired $h(s)$.

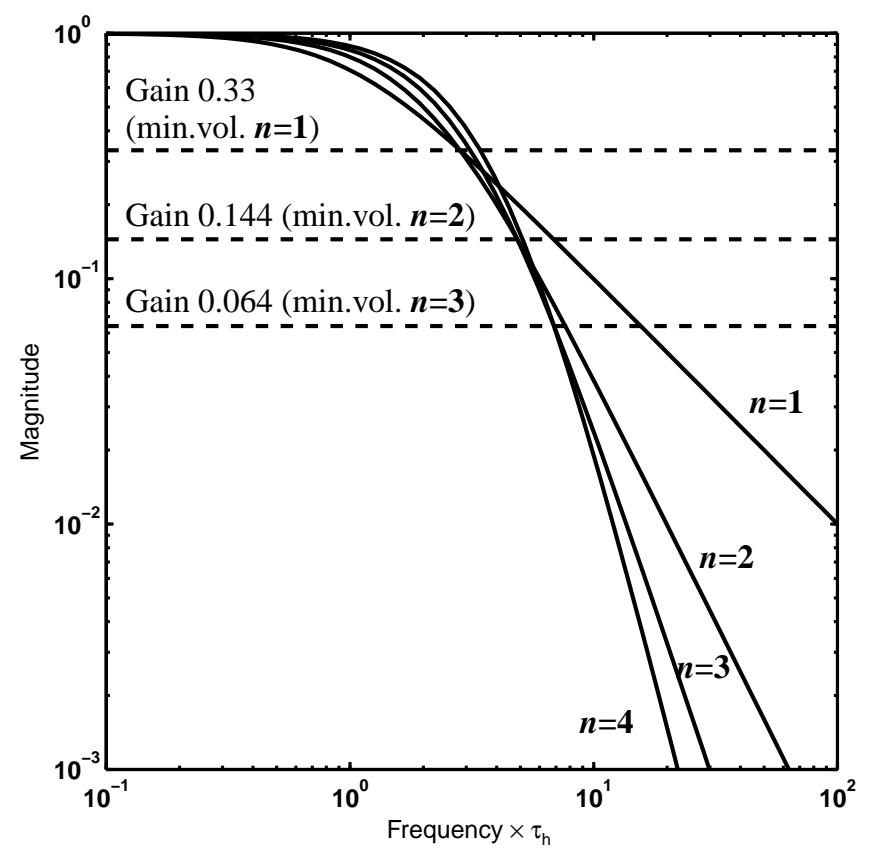

Figure 5: Frequency responses for $h(s)=1 /\left(\frac{\tau_{h}}{n} s+1\right)^{n}$.

Let us start with an uncontrolled plant without a buffer tank. The effect of the disturbance $d$ on the output $y$ is then

$$
y(s)=G(s) u(s)+G_{d_{0}}(s) d(s)
$$


To counteract the effect of the disturbances, we apply feedback control $(u=-K y$ ) (see Figure 6). The resulting closed-loop response becomes

$$
y(s)=S(s) G_{d_{0}}(s) d(s) ; \quad S=\frac{1}{1+G K}
$$

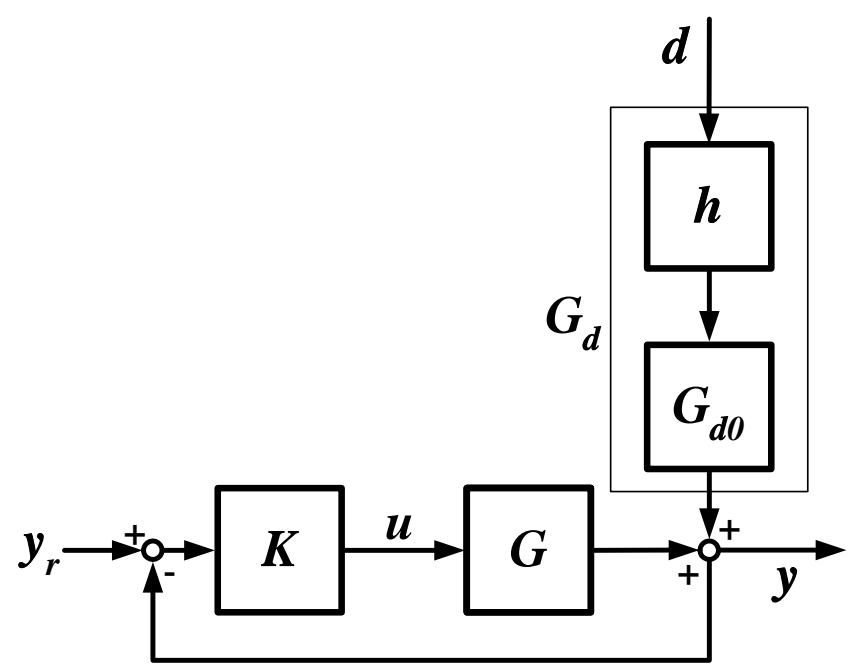

Figure 6: Feedback control system

With integral action in the controller, the sensitivity function $S$ approaches zero at low frequencies. However, at higher frequencies, the disturbance response, $\left|S(j \omega) G_{d_{0}}(j \omega)\right|$, may still be too large, and this is the reason for installing a buffer tank. The closed-loop response with a buffer tank is

$$
y(s)=S(s) \underbrace{G_{d_{0}}(s) h(s)}_{G_{d}(s)} d(s)
$$

which is acceptable if $\left|S G_{d_{0}} h\right|$ is sufficiently small at all frequencies. We need to quantify the term "sufficiently small", and we define it as "smaller than 1". More precisely, we assume that the variables and thus the model $\left(G_{d_{0}}\right)$ has been scaled such that

- The expected disturbance is less than $1(|d| \leq 1, \forall \omega)$

- The allowed output variation is less than $1(|y| \leq 1, \forall \omega)$

From (21) we see that to keep $|y| \leq 1$ when $|d|=1$ (worst-case disturbance), we must require

$$
\left|S(j \omega) G_{d 0}(j \omega) h(j \omega)\right| \leq 1 ; \forall \omega
$$

from which we can obtain the required $h(s)$. We illustrate the idea with an example.

Example 1 (continued) (Mixing process). Let $y=c_{0}, d=c_{A, \mathrm{f}}$, and $u=q_{B}$. Linearizing and scaling the model (3) then yields

$$
G_{d_{0}}(s)=\frac{10}{s+1} ; \quad S(s)=\frac{1}{1+\frac{0.5}{s} e^{-s}} ; \quad h(s)=\frac{1}{19 s+1}
$$


We here used for the scaling the following: expected variations in $c_{A}, \pm 20 \mathrm{~mol} / \mathrm{m}^{3}$; range for $q_{B}, \pm 0.5 \mathrm{~m}^{3} / \mathrm{min}$; allowed range for $c: \pm 1 \mathrm{~mol} / \mathrm{m}^{3}$.

In Figure 7 we plot the disturbance effects $\left|G_{d_{o}}\right|,\left|S G_{d_{0}}\right|$, and $\left|S G_{d_{0}} h\right|$ as functions of frequency. Originally (without any buffer tank or control), we have $\left|G_{d_{0}}\right|=10$ at lower frequencies. The introduction of feedback makes $\left|S G_{d_{0}}\right|<1$ at low frequencies, whereas adding the buffer tank brings $\left|S G_{d_{0}} h\right|<1$ also at intermediate frequencies.

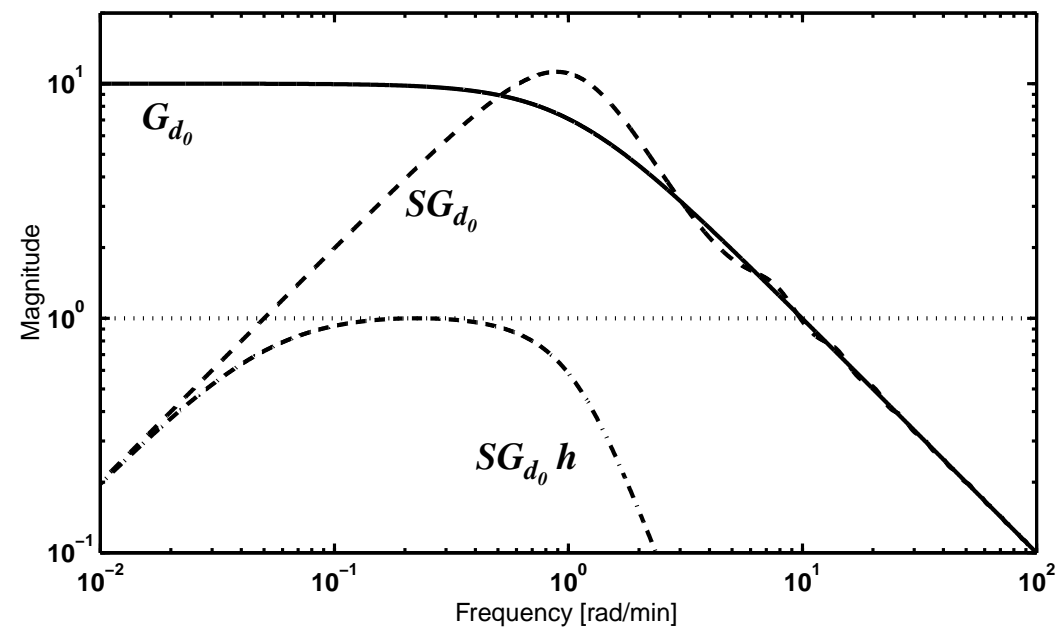

Figure 7: Original disturbance effect $\left(G_{d_{0}}\right)$, with feedback control $\left(S G_{d_{0}}\right)$ and with feedback control and a buffer tank $\left(S G_{d_{0}} h\right)$. A buffer tank with a residence time of 19 min is required to bring $\left|S(j \omega) G_{d_{0}}(j \omega) h(j \omega)\right|<1$ for all $\omega$.

In the following we will present methods for finding $h(s)$ based on the controllability requirement (22). There are two main cases:

S. Existing plant with an existing controller: The "counteracting" controller, $K(s)$, is already designed, so $S(s)$ is known. The "ideal" $h(s)$ is then simply the inverse of $S G_{d_{0}}$.

N. New plant: The "counteracting" controller, $K(s)$, is not known so $S(s)$ is not known. This is the typical situation during the design stage when most buffer tanks are designed.

In most cases we will choose $h(s)$ to be of the form $h(s)=1 /(\tau s+1)^{n}$.

\section{1 $S$ given (existing plant)}

We consider an existing plant where controller $K(s)$ is known. The task is to find $h(s)$ such that $|h(s)|<1 /\left|S G_{d_{0}}\right| ; \forall \omega$. Several approaches may be suggested.

S1. Graphical approach with $h(s)=1 /(\tau s+1)^{n}$ : This is done by selecting $h(s)=$ $1 /(\tau s+1)^{n}$ and adjusting $\tau$ until $|h(s)|$ touches $1 /\left|S G_{d_{0}}\right|$ at one frequency. As a starting point we choose the following:

(a) $n$ is the slope of $\left|S G_{d_{0}}\right|$ in a $\log -\log$ plot in the frequency area where $\left|S G_{d_{0}}\right|>1$.

(b) $\tau$ is the inverse of the frequency where $\left|S G_{d_{0}}\right|$ crosses one from below. 
S2 Numerical approach with $h(s)=1 /(\tau s+1)^{n}$ : With a given $n$ we find $\tau$ such that $|h|$ just touches $1 /\left|S G_{d_{0}}\right|$ by solving the following problem:

$$
\tau=\max _{\omega} \tau_{r e q}(\omega)
$$

where

$$
\tau_{r e q}(\omega)= \begin{cases}\frac{1}{\omega} \sqrt{\left|S(j \omega) G_{d_{0}}(j \omega)\right|^{2 / n}-1} ; & \left|S(j \omega) G_{d_{0}}(j \omega)\right|>1 \\ 0 ; & \text { otherwise }\end{cases}
$$

Because it is not practical to calculate $\tau_{r e q}(\omega)$ for all frequencies, we replace $\max _{\omega}$ with $\max _{\omega_{i}}$, where $\omega_{i} \in \Omega$, which is a finite set of frequencies from the range of interest. The calculation is explicit and fast, so a large number of frequencies can be used. (This approach was used to obtain $h(s)=1 /(19 s+1)$ in Figure 7.)

As illustrated in Example 2 (below), for $n>1$ one may save some volume with the following approach, which is more involved since it includes nonconvex optimization.

S3. Numerical approach with "free" $h(s)$ : We formulate a constrained optimization problem that minimizes the (total) volume of the buffer tank(s) subject to (22). As in the previous method, we formulate the optimization for a finite set of frequencies, $\Omega$, from the frequency range of interest.

(I) Quality disturbances: For $n$ mixing tanks

$$
h(s)=\frac{1}{\left(\tau_{1} s+1\right) \cdots\left(\tau_{n} s+1\right)}
$$

when the tanks are not necessarily equal. Because the flow rate is independent of the volumes $(\tau=V / q)$, we may minimize the total residence time (instead of minimizing the total volume) subject to (22):

$$
\begin{gathered}
\min _{\tau_{1}, \ldots, \tau_{n}} \tau_{1}+\cdots+\tau_{n} \\
\text { subject to } \\
\left|\left(\tau_{1} j \omega_{i}+1\right) \cdots\left(\tau_{n} j \omega_{i}+1\right)\right| \geq\left|S\left(j \omega_{i}\right) G_{d_{0}}\left(j \omega_{i}\right)\right| ; \omega_{i} \in \Omega
\end{gathered}
$$

where $\Omega$ is a set of frequencies. This is a single-input, single-output variant of a method proposed by Zheng and Mahajanam (1999).

(II) Flow-rate disturbances:

$$
h(s)=\frac{k(s, p)}{s+k(s, p)}
$$

where we have parametrized the level controller with the parameter vector $p$. We minimize subject to (22) the required tank volume (14):

$$
\begin{gathered}
\min _{p} V=\min _{p} \max _{\omega_{i} \in \Omega}\left|\frac{1}{j \omega_{i}+k\left(j \omega_{i}, p\right)}\right| \\
\text { subject to } \\
\left|S\left(j \omega_{i}\right) G_{d_{0}}\left(j \omega_{i}\right) \frac{k\left(j \omega_{i}, p\right)}{j \omega_{i}+k\left(j \omega_{i}, p\right)}\right| \leq 1 ; \omega_{i} \in \Omega
\end{gathered}
$$


Many controller formulations are possible, for example, the familiar PI(D) ( $D=$ derivative) controller or a state-space formulation. We here express the controller by a steadystate gain, $k_{s}, n_{Z}$ real zeros, and $n_{P}$ real poles:

$$
k(s, p)=k_{s} \frac{\left(T_{1} s+1\right)\left(T_{2} s+1\right) \cdots\left(T_{n_{Z}} s+1\right)}{\left(\tau_{1} s+1\right)\left(\tau_{2} s+1\right) \cdots\left(\tau_{n_{P}} s+1\right)}
$$

and thus $p=\left[k_{s}, T_{1}, \ldots, T_{n_{Z}}, \tau_{1}, \ldots, \tau_{n_{P}}\right]$.

With $n_{Z}=0$ and $n_{P}=1$ in (30) we get

$$
h(s)=\frac{1}{\tau^{2} s^{2}+2 \tau \zeta s+1}
$$

$\zeta<1$ does not give real time constants as the previous approaches. For a firstorder filter (with $k(s)=k_{s}$ and $h(s)=1 /(\tau s+1)$ ), there is no extra degree of freedom in the optimization, and we get the same result as that with (24).

Example 2 (Temperature control with flow-rate disturbance).

$$
\begin{aligned}
G_{d_{0}}(s) & =100 ; \quad G(s)=\frac{200}{100 s+1} e^{-s} \\
K_{\text {TIC }}(s) & =0.25 \frac{8 s+1}{8 s}
\end{aligned}
$$

This may represent the process in Figure 8, where two streams $A$ and $B$ are mixed, and we want to control the temperature (y) after the mixing point. Stream $A$ is heated in a heat exchanger,

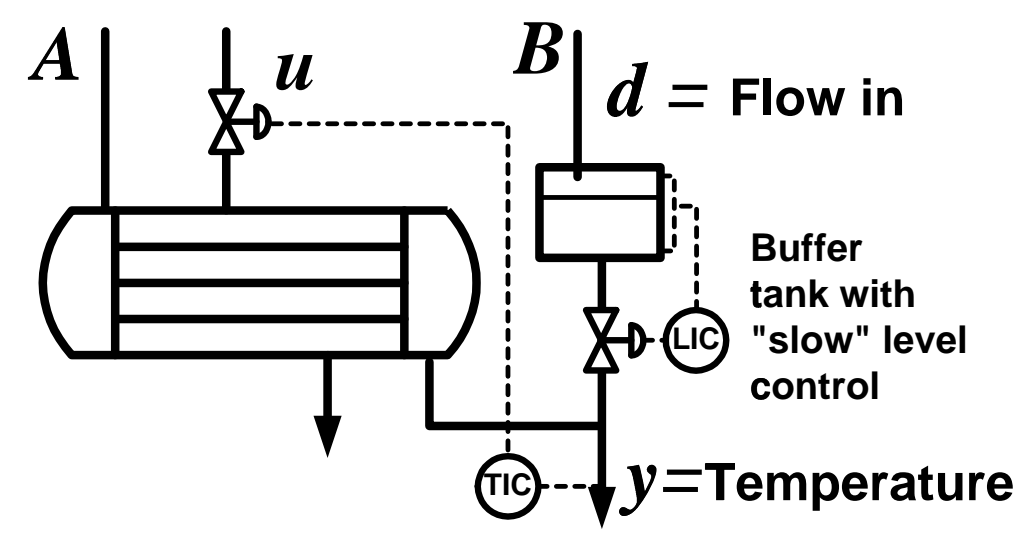

Figure 8: Temperature control with flow-rate disturbance

and the manipulated input, $u$, is the secondary flow rate in this exchanger. The disturbance, $d$, is variation from the nominal flow rate of $B . d, u$, and y are scaled as outlined above.

First consider the case without the buffer tank. Because $G_{d_{0}}=100$, the disturbance has a large impact on the output, and a temperature controller is certainly required. However, this is not sufficient because, as seen in Figure 9, $\left|S G_{d_{0}}\right|$ exceeds 1 at higher frequencies and it approaches 100 at high frequencies.

We thus need to install a buffer tank with averaging level control to dampen the flow-rate disturbance at higher frequencies. The slope of $\left|S G_{d_{0}}\right|$ is 2 after it has crossed 1, so one would expect that a second order $h(s)$ is the best. 


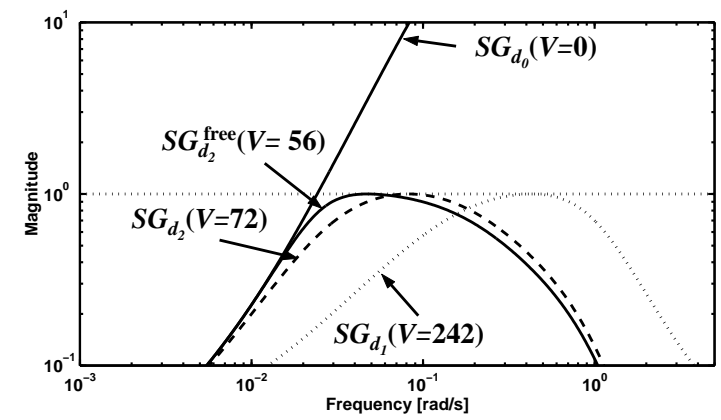

Figure 9: A buffer tank is needed for the temperature control problem: $\left|S G_{d_{0}}\right|>0$ for frequencies above $0.024 \mathrm{rad} / \mathrm{s}$. Comparison of $\left|S G_{d}\right|=\left|S G_{d_{0}} h\right|$ for designs 1,2 and 3 in Table 2.

Table 2: Buffer (surge) tank design procedure II (flow-rate disturbance) applied to the temperature control example

\begin{tabular}{|l|l|l|l|}
\hline Step & Design 1 & Design 2 & Design 3 \\
\hline 1. Numerical approach to obtain $h(s)$ & S2: $h(s)$ 1st order & S2: $h(s)$ 2nd order & S3(II): $h(s)$ 2nd order \\
\hline 2.1. Desired $h(s)$ (from Step 1) & $\frac{1}{242 s+1}$ & $\frac{1}{(36+1)^{2}}$ & $\frac{1}{1548 s^{2}+53.3 s+1}$ \\
\hline 2.2. Level controller, $k(s)$ & 0.0041 & $\frac{0.014}{18 s+1}$ & $\frac{0.019}{29 s+1}$ \\
\hline 2.3. $V(0) / q_{\text {in }}(0)$ & 242 & $2 \cdot 36=72$ & 56 \\
\hline 2.4. $V_{\text {tot }}$ & $242 \Delta q_{\max }$ & $72 \Delta q_{\max }$ & $56 \Delta q_{\max }$ \\
\hline
\end{tabular}

For the graphical approach $S 1$, we use $h(s)=1 /(\tau s+1)^{2} .\left|S G_{d 0}\right|$ crosses 1 at about frequency $0.024 \mathrm{rad} / \mathrm{s}$, corresponding to $\tau \approx 1 / 0.024=42$, and because this is a flow-rate disturbance (II), we have from Table 1 that $V_{\text {tot }}=2 \tau \Delta q_{\max } \approx 84 \Delta q_{\max }$. The required level controller is $k(s)=0.012 /(21 s+1)$.

For the more exact numerical approaches (S2 and S3), we consider three designs, and the results are given in Table 2. Design 1 (with $h(s)=1 /(\tau s+1)$ ) only requires a P level controller, but as expected, the required volume is large because $h(s)$ is first-order. Design $2\left(\right.$ with $\left.h(s)=1 /(\tau s+1)^{2}\right)$ gives a considerably smaller required volume. From design 3 (with $h(s)$ in $(31))$, the required volume is even smaller than with design 2 , as expected. Little is gained by increasing the order of $h(s)$ above 2.

In Figure 9 we plot the resulting $\left|S G_{d}\right|$ for the three designs, which confirms that they stay below 1 in magnitude at all frequencies. These results are further confirmed by the time responses to a unit step disturbance shown in Figure 10.

Buckley's method (Buckley, 1964) gives a residence time of $10 \mathrm{~min}$, which is much less than the minimum required residence time of about $56 \mathrm{~min}$ (see Table 2). The reason is that the disturbance needs to be reduced by a factor of 100, and not 10 as Buckley implicitly assumes.

\section{2 $S$ not given}

The requirement is that (22) must be fulfilled; that is, the buffer tank with transfer function $h(s)$ must be designed such that $\left|S G_{d_{0}} h\right| \leq 1$ at all frequencies. However, at the design stage the controller and thus $S$ is not known. Three approaches are suggested: 


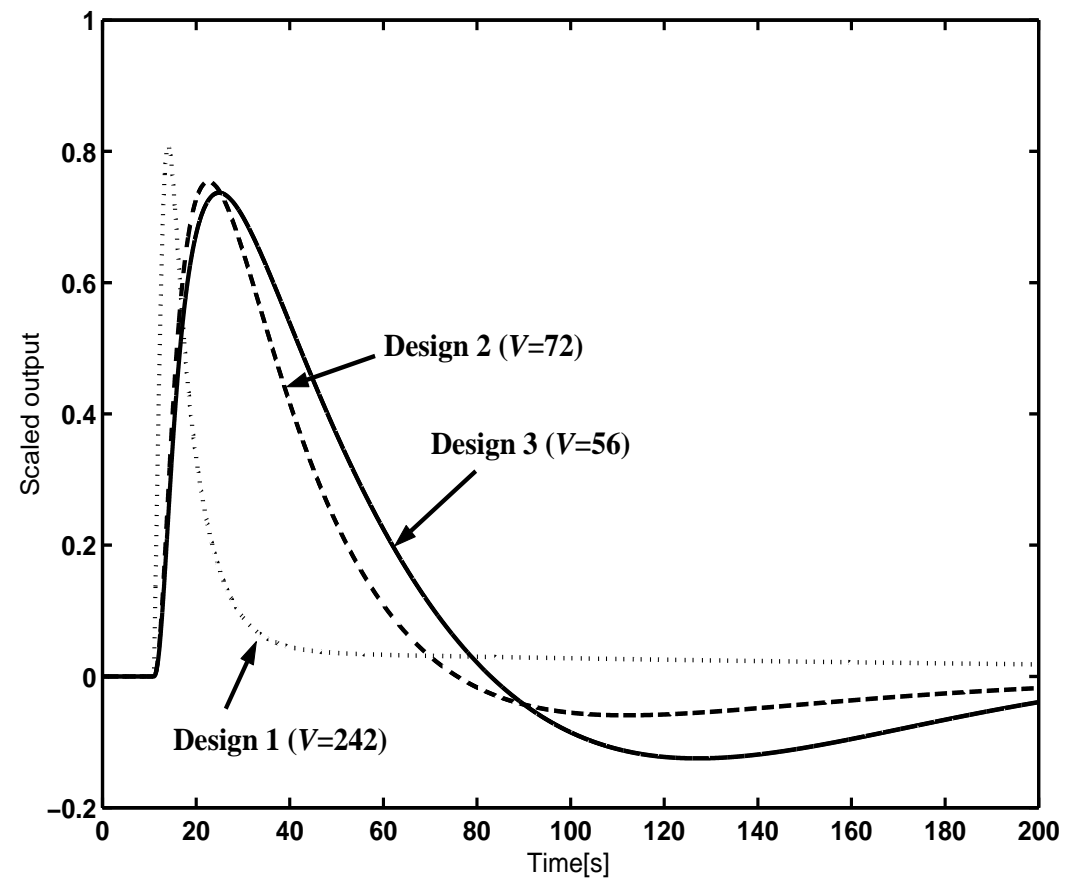

Figure 10: Temperature control with flow-rate disturbance: Response in the scaled output to a unit step in the disturbance (flow rate) with different tank sizes and level controllers (Table 2).

N1. Shortcut approach: The requirement (22) must, in particular, be satisfied at the bandwidth frequency $\omega_{B}$ where $|S|=1$, and this gives the (minimum) requirement

$$
\underbrace{\left|G_{d_{0}}\left(j \omega_{B}\right)\right|}_{f}\left|h\left(j \omega_{B}\right)\right| \leq 1 \Longleftrightarrow\left|h\left(j \omega_{B}\right)\right| \leq 1 / f
$$

In Skogestad and Postlethwaite (1996, p. 173-4) it is suggested that $\omega_{B} \leq \frac{1}{\theta_{\text {eff }}}$, where $\theta_{\text {eff }}$ is the effective delay around the feedback loop. However, to get acceptable robustness, we here suggest to use a somewhat lower value

$$
\omega_{B} \approx \frac{1}{2 \theta_{\mathrm{eff}}}
$$

Skogestad (2003) proposes the following simple rule for estimating $\theta_{\text {eff }}$ :

$$
\theta_{\text {eff }}=\theta+\tau_{z}+\frac{\tau_{j}}{2}+\sum_{i>j} \tau_{i} ; \quad \begin{aligned}
& j=2 \text { for PI-control } \\
& j=3 \text { for PID-control }
\end{aligned}
$$

where $\theta$ is the delay, $\tau_{z}=1 / z$ is the inverse of a right half-plane zero $z$, and $\tau_{i}$ is the time lag (time constant) number $i$ ordered by size so that $\tau_{1}$ is the largest time constant.

We now assume $h(s)=1 /(\tau s+1)^{n}$, use $\omega_{B}=1 /\left(2 \theta_{\text {eff }}\right)$, and solve (34) to get

$$
\tau \geq 2 \theta_{\text {eff }} \sqrt{f^{2 / n}-1}
$$

where $f \stackrel{\text { def }}{=}\left|G_{d_{0}}\left(j \frac{1}{2 \theta_{\text {eff }}}\right)\right|$. Alternatively, Figure 5 may be used for a given $n$ to read off the normalized frequency $v=\omega \tau_{h}$ where $|h(j v)|=1 / f$, and the required $\tau$ for each tank is then $\tau=v /\left(n \omega_{B}\right)$. 
N2. Numerical approach based on preliminary controller design: The above shortcut method only considers the frequency $\omega_{B}$. To get a more exact design, we must consider all frequencies, and a preliminary controller design is needed. This approach consists of two steps:

N2a. Find a preliminary controller for the process, and from this, obtain $S(s)$.

N2b. Use one of the approaches S1, S2, or S3 from section 4.1.

For step N2a, we have used the method of Schei (1994), where we maximize the lowfrequency controller gain $K_{I}=k_{c} / \tau_{I}$, subject to a robustness restriction (maximum value on the peak of $S$ ):

$$
\begin{gathered}
\min \tau_{I} / k_{c} \\
\text { subject to } \\
\left|S\left(j \omega_{i}\right)\right|<M_{S} ; \omega_{i} \in \Omega \text { and } S \text { stable }
\end{gathered}
$$

where for a PI controller $K(s)=k_{c}\left(\tau_{I} s+1\right) /\left(\tau_{I} s\right)$. Compared to the optimization problem that Schei uses, we have added the constraint that $S$ is stable. This is implemented by requiring the eigenvalues of $\tilde{S}$ to be in the left half-plane, where $\tilde{S}$ is obtained from $S$ by replacing the delay with a Padé approximation. To obtain a robust design, $M_{S}$ should be chosen low, typically $1.6-2$. With this controller design, we then use one of the methods S1-S3 to design the buffer tank.

N3. Numerical approach with a simultaneous controller and buffer tank design. A more exact approach is to combine the controller tuning and the buffer tank design optimization into one problem. For (I) quality disturbances, the optimization problem may be formulated as an extension of (27):

$$
\begin{gathered}
\min _{\tau_{1}, \ldots, \tau_{n}, p_{K}} \tau_{1}+\cdots+\tau_{n} \\
\text { subject to } \\
\left|\left(\tau_{1} j \omega_{i}+1\right) \cdots\left(\tau_{n} j \omega_{i}+1\right)\right| \geq\left|S\left(j \omega_{i}, p_{K}\right) G_{d_{0}}\left(j \omega_{i}\right)\right| ; \omega_{i} \in \Omega \\
\left|S\left(j \omega_{i}, p_{K}\right)\right|<M_{S} ; \omega_{i} \in \Omega \\
S\left(p_{K}\right) \text { stable }
\end{gathered}
$$

where $p_{K}$ is the controller parameter vector for $K(s)$. Likewise for (II) flow-rate disturbances, we get from (29):

$$
\begin{gathered}
\min _{p, p_{K}} V=\min _{p, p_{K}} \max _{\omega_{i} \in \Omega}\left|\frac{1}{j \omega_{i}+k\left(j \omega_{i}, p\right)}\right| \\
\text { subject to } \\
\left|S\left(j \omega_{i}, p_{K}\right) G_{d_{0}}\left(j \omega_{i}\right) \frac{k\left(j \omega_{i}, p\right)}{j \omega_{i}+k\left(j \omega_{i}, p\right)}\right| \leq 1 ; \omega_{i} \in \Omega \\
\left|S\left(j \omega_{i}, p_{K}\right)\right|<M_{S} ; \omega_{i} \in \Omega \\
S\left(p_{K}\right) \text { stable }
\end{gathered}
$$

where $p$ is the controller parameter vector for the level controller $k(s)$, which enters in $h(s)$, and $p_{K}$ is the controller parameter vector for the feedback controller $K(s)$, which 
enters in $S(s)$. To ensure effective integral action in $K$, these optimization problems must be extended by a constraint; for example, if $K(s)$ is a PI controller, a maximum value must be put on the integral time.

Example 2 (continued) (Temperature control with flow-rate disturbance (II))

$$
G_{d_{0}}(s)=100 ; \quad G(s)=\frac{200}{100 s+1} e^{-s}
$$

The available information of the process is given by (41), and we assume that the controller is not known. The delay is $\theta=1 \mathrm{~s}$. We get the following results:

N1. The shortcut approach yields $\left(\omega_{B}=0.5 \mathrm{rad} / \mathrm{s}\right.$ and $f=\left|G_{d_{0}}\right|=100$ for all $\left.\omega\right)$ from (37) (or Figure 5) the following:

- First-order filter $(n=1): V_{\text {tot }}=200 \Delta q_{\max }$.

- Second-order filter $(n=2): V_{\text {tot }}=40 \Delta q_{\max }$.

N2. The Schei tuning in (38) followed by the optimal design (29) yields for a second order $h(s)\left(n_{Z}=0\right.$ and $\left.n_{P}=1\right)$ the following:

- $M_{S}=1.6: V_{t o t}=52 \Delta q_{\max }$.

- $M_{S}=2: V_{t o t}=39 \Delta q_{\max }$.

N3. Simultaneous controller tuning and optimal design (40) yields with second-order $h(s)$ $\left(n_{Z}=0\right.$ and $\left.n_{P}=1\right)$ the following:

- $M_{S}=1.6: V_{t o t}=52 \Delta q_{\max }($ as for method $N 2)$

- $M_{S}=2: V_{t o t}=39 \Delta q_{\max }$ (as for method $\left.N 2\right)$

Note that $M_{S}=1.6$ gives more robust (and "slow") controller tunings than $M_{S}=2$ and therefore requires a larger tank volume. The smallest achievable tank volume with a second-order filter is $V_{\text {tot }}=27 \Delta q_{\max }$ (found with method $N 3$ with $M_{S}$ free). Methods $N 2$ and N3 yield almost identical results for this example. The shortcut method N1 also gives a tank volume very similar to that found with $M_{S}=2$.

\section{Before or after?}

If the buffer tank is placed upstream of the process, the disturbance itself is dampened before entering the process. If it is placed downstream of the process, the resulting variations in the product are dampened. The control properties are mainly determined by the effect of input $u$ on output $y$ (as given by the transfer function $G$ ). An upstream buffer tank has no effect on $G$, and also a downstream buffer tank has no effect on $G$ provided we keep the original measurement. On the other hand, placement "inside" the process normally affects $G$. In the following we list some points that may be considered when choosing the placement. We assume that we prefer to have as few and small buffer tanks as possible (sometimes other issues come into consideration, like differences in cost due to different pressure or risk of corrosion, but this is not covered). 
1. In a "splitting process", the feed flow is split into two or more flows (Figure 11(a)). One common example is a distillation column. To reduce the number of tanks, it will then be best to place the buffer tank at the feed (upstream placement). An exception is if only one of the product streams needs to be dampened, in which case a smaller product tank can be used because each of the product streams are smaller than the feed stream.

2. In a "mixing" process, two or more streams are mixed into one stream (Figure 11(b)). To reduce the number of tanks, it is here best with a downstream placement. An exception is if we only have disturbances in one of the feed streams because the feed streams are smaller than the product stream, leading to a smaller required size.

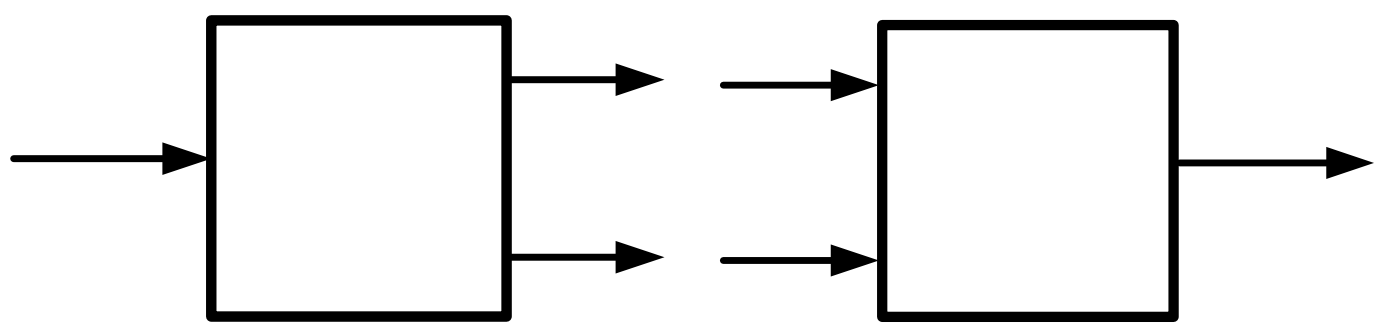
(a) A splitting process
(b) A mixing process

Figure 11: Two types of processes

3. An advantage of a downstream placement is that a downstream buffer tank dampens all disturbances, including disturbances in the control inputs. This is not the case with upstream tanks, which only dampen disturbances entering upstream of the tank.

4. An advantage of an upstream placement is that the process stays closer to its nominal operation point and thus simplifies controller tuning and makes the response more linear and predictable (see Example 3).

5. An advantage of the "inside" placement is that it may be possible to avoid installation of a new tank by making use of an already planned or existing unit, for example, by increasing the size of a chemical reactor.

6. A disadvantage with placing the buffer tank inside or downstream of the process is that the buffer tank then may be within the control loop, and the control performance will generally be poorer. Also, its size will effect the tuning, and the simultaneous approach (N3) is recommended. For the downstream placement, these problems may be avoided if we keep the measurement before the buffer tank, but then we may need an extra measurement in the buffer tank to get a more representative value for the final product.

Example 3 (Distillation column). We apply the methods from section 4.1 to a distillation column and compare the use of a single feed tank with the use of two product tanks (Figure 12). We consider a distillation column with 40 stages (the linearized model has 82 states; see column A from (Skogestad and Postlethwaite, 1996, p.425)). The disturbances to the column are feed flow rate and composition $\left(d_{1}=F\right.$ and $\left.d_{2}=z_{F}\right)$, and the outputs are the mole 
fractions of the component in top and bottom products, respectively $\left(y_{1}\right.$ and $\left.y_{2}\right)$. The manipulated variables are the reflux and the boilup $\left(u_{1}=L\right.$ and $\left.u_{2}=V\right)$. The variables have been scaled so that a variation of $\pm 30 \%$ in the feed flow rate corresponds to $d_{1}= \pm 1$ and a variation of $\pm 10 \%$ in the feed composition corresponds to $d_{2}= \pm 1$. A change in the top and bottom product composition of \pm 0.01 mole fraction units corresponds to a change \pm 1 in $y_{1}$ and $y_{2}$. Decentralized PI controllers are used to control the compositions. In the top, $K_{1}(s)=6.84(20 s+1) /(20 s)$, and in the bottom, $K_{2}=5.46(20 s+1) /(20 s)$. There is $a$ delay of $10 \mathrm{~min}$ in each loop, which we represent with fifth-order Padé approximations in the linear model. Nominally, the feed flow rate is $1 \mathrm{~m}^{3} / \mathrm{min}$, and the top and bottom concentrations are 0.99 and 0.01 , respectively.

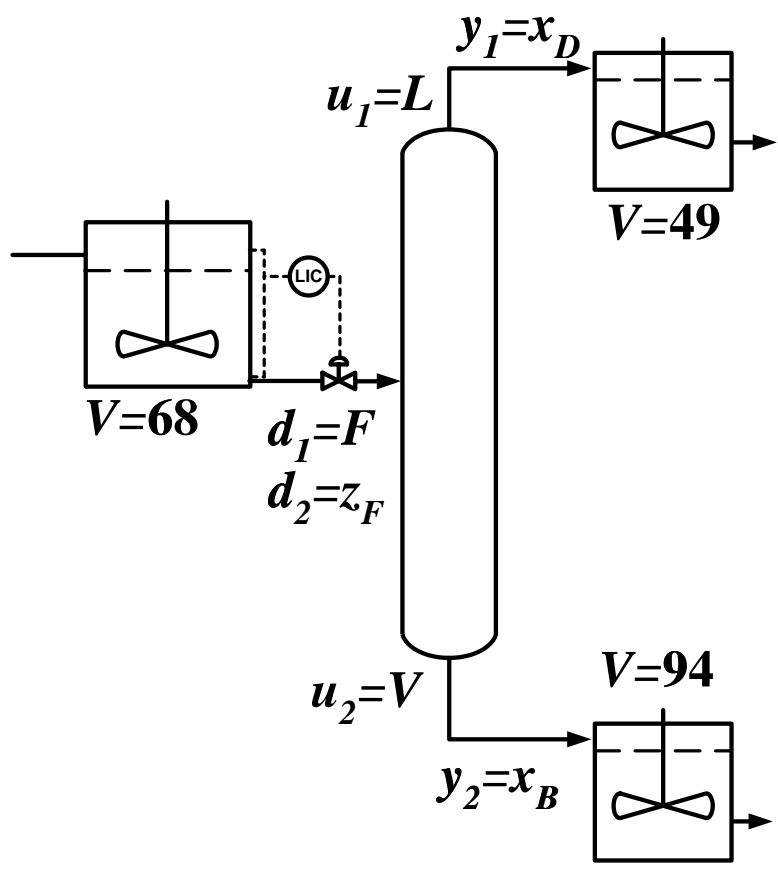

Figure 12: Distillation column with either one feed surge tank or two product mixing tanks to dampen disturbances.

The holdup in the reflux and the boiler are controlled with $P$ controllers (with gain 10) by the top and bottom product streams, respectively.

We consider the effect of the flow-rate disturbance, $d_{1}$. The closed-loop gains from $d_{1}$ to $y_{1}$ and $y_{2}$ without any buffer tank, $\left|S G_{d_{10}}\right|$ and $\left|S G_{d_{20}}\right|$ are shown with solid lines in Figure 13. The gains are both above 1 at intermediate frequencies, so our purity requirements will not be fulfilled, unless we install a buffer tank.

Upstream placement (feed surge tank). $S$ is known, and with $n=1,(24)$ in method $S 2$ yields $\tau=114 \mathrm{~min}$. The resulting $\left|S G_{d_{1}}\right|$ and $\left|S G_{d_{2}}\right|$ are shown with dashed lines, and we see that $\left|S G_{d_{2}}\right|$ just hits 1 (as expected). $1 /|h|$ is also plotted (dash-dotted) to indicate the limiting frequency, which is not at the maximum of $\left|S G_{d_{20}}\right|$, but at a lower frequency "shoulder". Following design procedure II, we now get the following:

$2.1 h(s)=1 /(114 s+1)$

2.2 The required level controller for the buffer tank is $k(s)=1 / 114=0.0088$ 
$2.3 V(0) / q_{\text {in }}(0)=\tau=114$

$2.4 V_{t o t}=\tau \Delta q_{\max }=114 \mathrm{~min} \cdot 2 \cdot 0.3 \mathrm{~m}^{3} / \min =68 \mathrm{~m}^{3}$.

Comment: Since the slope of $\left|S G_{d_{20}}\right|$ is less that 1 around the limiting frequency, higher order filters will increase the volume demand. For example, with $n=2$, (24) gives $\tau=$ $76.6 \mathrm{~min}$, and $V_{\text {tot }}=2 \tau \Delta q_{\max }=91.9 \mathrm{~m}^{3}$.

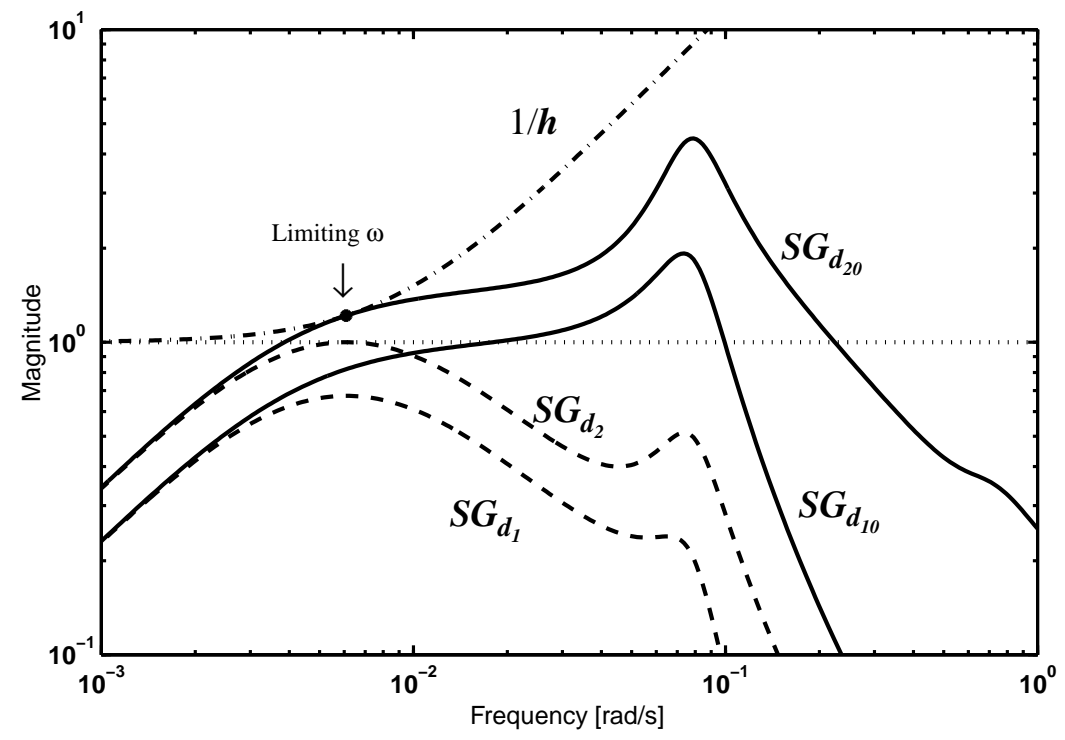

Figure 13: Feed flow disturbance for the distillation column: $\left|S G_{d_{10}}\right|$ and $\left|S G_{d_{20}}\right|$ (for top and bottom) are both above 1 (solid line). A feed tank with averaging level control, $h(s)=$ $1 /(114 s+1)$, brings the disturbance gain to both top and bottom below 1 (dashed). Note that $1 / h(s)$ is just touching $\left|S G_{d_{20}}\right|$.

Downstream placement (product mixing tank). Because both $\left|S G_{d_{10}}\right|>1$ and $\left|S G_{d_{20}}\right|>$ 1 at some frequencies, we must apply one mixing tank for each of the two products. When we designed the feed tank, we had to consider the worst of $\left|S G_{d_{10}}\right|$ and $\left|S G_{d_{20}}\right|$, but now we may consider $\left|S G_{d_{10}}\right|$ for the top product and $\left|S G_{d_{20}}\right|$ for the bottom product. With $n=1$, (24) yields $23 \mathrm{~min}$ for the top buffer tank and as before $114 \mathrm{~min}$ for the bottom tank. The corresponding volumes are $23 \cdot 0.5=11.5 \mathrm{~m}^{3}$ (top) and $114 \cdot 0.5=57 \mathrm{~m}^{3}$ (bottom), which gives a total volume of $69 \mathrm{~m}^{3}$, which is the same as that for the feed tank. However, the feed tank placement is preferred because we then need only one tank.

Nonlinear simulations. The above design is based on a linearized model, and (as expected) the feed tank placement is further justified if we consider a nonlinear model because the column is then less perturbed from its nominal state. This is illustrated by the simulations in Figures 14, 15 and 16. If the buffer tanks are placed downstream, the nonlinear response deviates considerably from the linear response, and the tanks designed by linear analysis are too small. By trial and error with disturbance step simulations on the nonlinear model, we find that $\tau_{t}=98 \mathrm{~min}$ and $\tau_{b}=188 \mathrm{~min}$ are needed for the top and bottom product tanks. This gives a total volume of $143 \mathrm{~m}^{3}$, considerably larger than the required feed tank of $68 \mathrm{~m}^{3}$.

In conclusion, an upstream feed tank with a P controller (averaging level control) proves best for this example. The example also illustrates that for nonlinear processes the buffer tank design methods that we have proposed are most reliable for the design of upstream buffer tanks. 


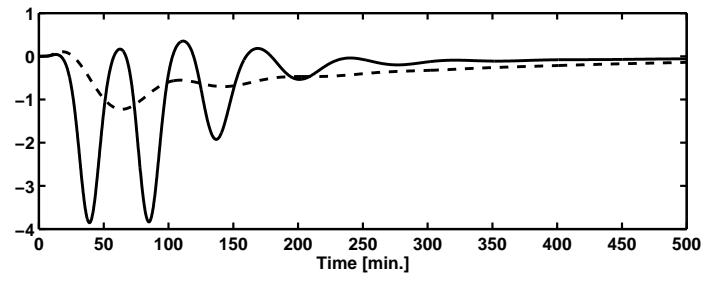

(a) Output $y_{1}$. Nonlinear simulation (solid) and linear simulation (dashed).

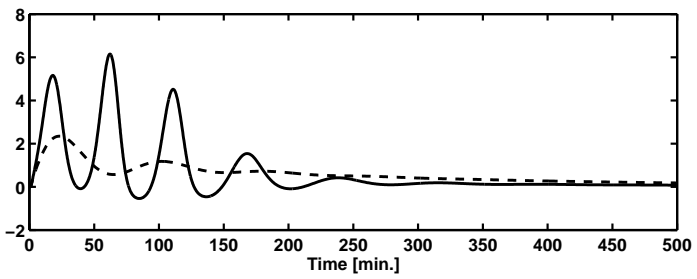

(b) Output $y_{2}$. Nonlinear simulation (solid) and linear simulation (dashed).

Figure 14: Distillation example with no buffer tanks installed. The control system is not able to handle the disturbance. There is a large deviation between nonlinear and linear simulation.

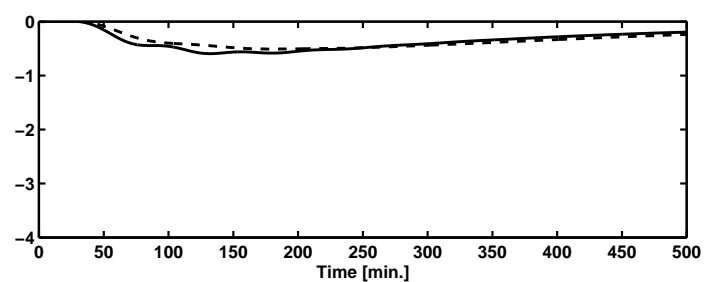

(a) Output $y_{1}$. Nonlinear simulation (solid) and linear simulation (dashed).

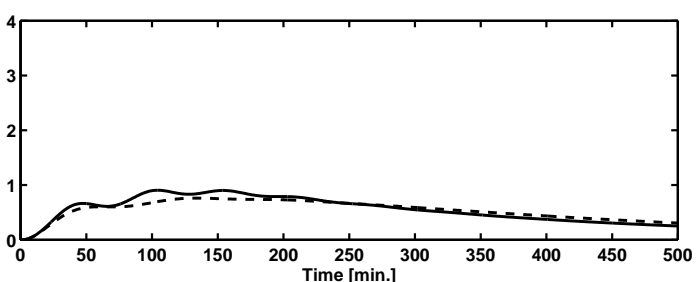

(b) Output $y_{2}$. Nonlinear simulation (solid) and linear simulation (dashed).

Figure 15: Distillation example with a feed tank of $68 \mathrm{~m}^{3}$. Both outputs stay within \pm 1 , and the nonlinear simulation is close to the linear one.

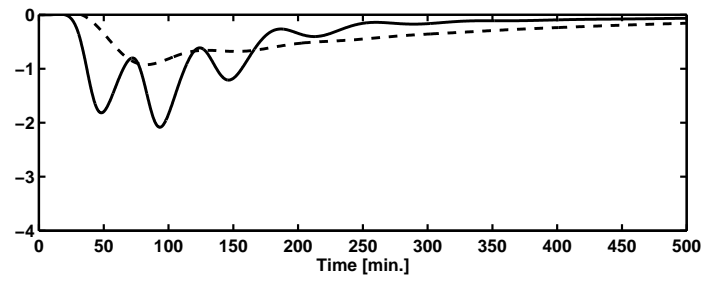

(a) Output $y_{1}$. Nonlinear simulation (solid) and linear simulation (dashed).

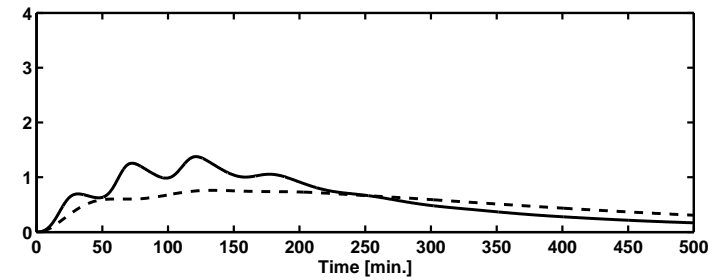

(b) Output $y_{2}$. Nonlinear simulation (solid) and linear simulation (dashed).

Figure 16: Distillation example with product tanks at the top $\left(11.5 \mathrm{~m}^{3}\right)$ and at the bottom $\left(57 \mathrm{~m}^{3}\right)$. The outputs deviate from \pm 1 in the nonlinear simulations. 
For (highly) nonlinear processes, the results should, if possible, be checked with simulations on a nonlinear model.

\section{Conclusions}

The controlled variables $(y)$ must be kept within certain limits despite disturbances $(d)$ entering the process. High-frequency components of disturbances are dampened by the process itself, while low-frequency components, e.g., the long-term effect of a step, are handled by the control system. There are, however, always limitations in how quickly a control system can react, for example, as a result of delays. Thus, for some processes there is a frequency range where the original process and the controller do not dampen the disturbance sufficiently. In this paper we introduce methods for designing buffer tanks based on this insight. The methods consist of two steps:

Step 1. Find the required transfer function $h(j \omega)$ such that $\left|S(j \omega) G_{d_{0}}(j \omega) h(j \omega)\right|<1 ; \forall \omega$ (with scaled variables). The methods for this have been divided into two groups depending on whether the control system for the process is already designed (methods S1-S3) or not (methods N1-N3). The shortcut methods (S1/S2 or N1), supplemented with nonlinear simulations, are recommended for most practical designs.

Step 2. Design a buffer tank that realizes this transfer function $h(s)$. For a first-order transfer function, $h(s)=1 /(\tau s+1)$, we have the following:

I. Quality disturbances Install a mixing tank with volume $V=q \tau$, where $q$ is the nominal flow rate.

II. Flow-rate disturbances Install a tank with averaging level control with gain $k(s)=$ $1 / \tau$ and volume $V=\tau \Delta q_{\max }$ where $\Delta q_{\max }$ is the expected range (from minimum to maximum) in the flow-rate variation.

Sometimes a higher-order $h(s)$ is preferable, in which case we need (I) for quality disturbances more than one mixing tanks and (II) for flow-rate disturbances a more complicated level controller $k(s)$ (with lags) (see Table 1).

\section{References}

Buckley, P. S. (1964). Techniques of Process Control. John Wiley \& Sons, Inc.. New York.

Faanes, A. and S. Skogestad (2002). pH-neutralization: Integrated process and control design. Submitted to Comput. Chem. Engng.

Harriott, P. (1964). Process Control. McGraw-Hill. New York.

Hiester, A. C., S. S. Melsheimer and E. F. Vogel (1987). Optimum size and location of surge capacity in continuous chemical processes. AIChe Annual Meet, Nov. 15-20, 1987, paper86c.

Lieberman, N. P. (1983). Process Design for Reliable Operations. Gulf Publishing Company. Houston. 
Ludwig, E. E. (1977). Applied Process Design for Chemical and Petrochemical Plants. Vol. 1. Gulf Publishing Company. Houston.

Marlin, T. E. (1995). Process Control. Designing Processes and Control Systems for Dynamic Performance. McGraw-Hill, Inc.. New York.

McDonald, K. A., T. J. McAvoy and A. Tits (1986). Optimal averaging level control. AIChE J. 32(1), 75-86.

McMillan, G. K. (1984). pH Control. Instrument Society of America. Research Triangle Park, NC, USA.

Sandler, H. J. and E. T. Luckiewicz (1987). Practical Process Engineering. McGraw-Hill Book Company. New York.

Schei, T. S. (1994). Automatic tuning of PID controllers based on transfer function estimation. Automatica 30(12), 1983-1989.

Shinskey, F. G. (1973). pH and pIon Control in Process and Waste Streams. John Wiley \& Sons. New York.

Shinskey, F. G. (1996). Process Control Systems - Application, Design, and Tuning, 4th Ed.. McGraw-Hill Inc., New York.

Shunta, J. P. and W. Fehervari (1976). Nonlinear control of liquid level. Instrum. Technol. pp. 43-48.

Sigales, B. (1975). How to design reflux drums. Chem. Eng. 82(5), 157-160.

Skogestad, S. (2003). Simple analytic rules for model reduction and PID controller tuning. J. Proc. Contr. 13(4), 291-309.

Skogestad, S. and I. Postlethwaite (1996). Multivariable Feedback Control. John Wiley \& Sons. Chichester, New York.

Ulrich, G. D. (1984). A Guide to Chemical Engingeering Process Design and Economics. John Wiley \& Sons. New York.

Walas, S. M. (1987). Rules of thumb, selecting and designing equipment. Chem. Eng. 94(4), 75-81.

Walsh, S. (1993). Integrated Design of Chemical Waste Water Treatment Systems. PhD thesis. Imperial College, UK.

Watkins, R. N. (1967). Sizing separators and accumulators. Hydrocarbon Processing 46(11), 253-256.

Wells, G. L. (1986). The Art of Chemical Process Design. Elsevier. Amsterdam.

Zheng, A. and R. V. Mahajanam (1999). A quantitative controllability index. Ind. Eng. Chem. Res. 38, 999-1006. 